\title{
Meta-analysis of randomized controlled trials only exploring the role of single incision laparoscopic surgery versus conventional multiport laparoscopic surgery for colorectal resections
}

\author{
Madhusoodhana Hebbar ${ }^{1}$, Waleed Riaz ${ }^{2}$, Parv Sains ${ }^{2}$, Mirza Khurrum Baig ${ }^{1}$, Muhammad Shafique Sajid ${ }^{2}$ \\ ${ }^{1}$ Department of General and Laparoscopic Colorectal Surgery, Western Sussex Hospitals NHS Foundation Trust, Worthing Hospital, Worthing, \\ West Sussex, BN11 2DH, UK; ${ }^{2}$ Department of General and Laparoscopic Colorectal Surgery, Brighton \& Sussex University Hospitals NHS Trust, \\ The Royal Sussex County Hospital, Brighton, West Sussex, BN2 5BE, UK \\ Contributions: (I) Conception and design: M Hebbar, MS Sajid; (II) Administrative support: P Sains, MK Baig; (III) Provision of study materials or \\ patients: M Hebbar, W Riaz, P Sains, MS Sajid; (IV) Collection and assembly of data: M Hebbar, W Riaz; (V) Data analysis and interpretation: M \\ Hebbar, MS Sajid, MK Baig; (VI) Manuscript writing: All authors; (VII) Final approval of manuscript: All authors. \\ Correspondence to: Madhusoodhana Hebbar. Department of Surgery, Worthing Hospital, Lyndhurst Road, Worthing, BN11 2DH, UK. \\ Email: krmhebbar@gmail.com.
}

\begin{abstract}
Background: The objective of this article is to evaluate the surgical outcomes in patients undergoing single incision laparoscopic surgery (SILS) versus conventional multi-incision laparoscopic surgery (MILS) for colorectal resections.

Methods: The data retrieved from the published randomized controlled trials (RCTs) reporting the surgical outcomes in patients undergoing SILS versus MILS for colorectal resections was analysed using the principles of meta-analysis. The combined outcome of dichotomous data was represented as risk ratio (RR) and continuous data was shown as standardized mean difference (SMD).

Results: Five RCTs on 525 patients reported the colorectal resections by SILS versus MILS technique. In the random effects model analysis using the statistical software Review Manager 5.3, the operation time (SMD, 0.20; 95\% CI, -0.11 to 0.52; $\mathrm{z}=1.28 ; \mathrm{P}=0.20$ ), length of in-patient stay (SMD, $-0.18 ; 95 \% \mathrm{CI},-0.51$ to $0.14 ; \mathrm{z}=1.10 ; \mathrm{P}=0.27$ ) and lymph node harvesting (SMD, 0.09; 95\% CI, -0.14 to $0.33 ; \mathrm{z}=0.76 ; \mathrm{P}=0.45$ ) were comparable between both techniques. Furthermore, post-operative complications (RR, 1.00; 95\% CI, 0.65-1.54; $\mathrm{z}=0.02 ; \mathrm{P}=0.99)$, post-operative mortality, surgical site infection rate (RR, 3.00; 95\% CI, 0.1370.92; $\mathrm{z}=0.68 ; \mathrm{P}=0.50)$, anastomotic leak rate (RR, 0.43; 95\% CI, 0.11-1.63; $\mathrm{z}=1.24 ; \mathrm{P}=0.21)$, conversion rate $(\mathrm{P}=0.13)$ and re-operation rate $(\mathrm{P}=0.43)$ were also statistically similar following SILS and MILS.
\end{abstract}

Conclusions: SILS failed to demonstrate any superiority over MILS for colorectal resections in all postoperative surgical outcomes.

Keywords: Single incision laparoscopic surgery (SILS); multi-incision laparoscopic surgery (MILS); colorectal cancer; diverticular disease; colorectal resections

Received: 26 April 2018; Accepted: 08 May 2018; Published: 29 May 2018.

doi: $10.21037 / \operatorname{tgh} .2018 .05 .05$

View this article at: http://dx.doi.org/10.21037/tgh.2018.05.05

\section{Introduction}

Approximately 95,000 new cases of colon cancer and 40,000 new cases of rectal cancer were diagnosed in the USA in 2017 (1). Colonic diverticulosis has the highest prevalence in United States, Europe and Australia, where approximately 50\% of the population over the age of 60 years have diverticulosis $(2,3)$. Surgery is the main treatment for colorectal cancer and complicated colonic diverticular disease. Laparoscopic surgery is considered a gold standard treatment for colorectal resection (4-9) due to several advantages such as faster recovery of 
bowel function, early feeding; lower post-operative pain, early mobilization and short hospital stay (10-12). Furthermore, the laparoscopic colorectal resections also offer similar long-term oncologic and survival outcomes as compared to open surgery (5,9,13-16). The clinical benefits of laparoscopic surgery seem to be due to the small incision in the abdominal wall, reduced bowel manipulation and thereby reduced surgical trauma (17).

Conventional laparoscopic surgery uses 3 to 6 ports of varying diameter at different location in the abdominal wall and a specimen extraction incision, with each port/ incision adding to surgical trauma related morbidity like pain, risk of bleeding, hernia and intra-peritoneal organ injury $(18,19)$. To reduce these risks and to maximize the clinical benefits, single incision laparoscopic surgery (SILS) was developed. Published meta-analyses have supported the safety and efficacy of this innovative approach of SILS for appendicectomy and cholecystectomy $(20,21)$. Potential benefits of SILS include better cosmesis, patient satisfaction, less post-operative pain and faster recovery. However, it increases the difficulty of the operation due to technical challenges of poor triangulation, instrument crowding and inadequate counter-traction (22-31). In spite of these obstacles, by using advanced technology several renowned colorectal centers in the world have reported the use of SILS for colorectal resections (23,32-38). Previously published systematic review and meta-analysis comparing the effectiveness of SILS versus traditional multi-incision laparoscopic surgery (MILS) have shown safety and feasibility of SILS (39-42) but these meta-analyses included non-randomized and retrospective comparative studies with significant bias and heterogeneity in study population. Hence the strength of clinical evidence from these metaanalyses was weaker and biased.

The objective of this article is to evaluate the surgical outcomes in patients undergoing SILS versus MILS for colorectal resections using the principles of meta-analysis and analyse data from published randomized controlled trials (RCTs) to establish and validate best possible evidence to date.

\section{Methods}

\section{Electronic data base search}

Medline (via PubMed), Embase, Scopus, Cochrane Colorectal Cancer Group (CCCG) Controlled Trial Register, the Cochrane Central Register of Controlled Trials (CENTRAL) in the Cochrane Library and Science
Citation Index Expanded were explored until December 2017 to find relevant published RCTs. The search terms were constructed based on patients, interventions/ comparators, and outcomes as follows:

* Patients: colorectal cancer, colon cancer, rectal cancer, diverticular disease;

* Intervention/comparator: SILS, MILS, laparoscopic resection, minimal invasive surgery;

* Outcomes: duration of operation, length of stay in hospital, lymph nodes harvesting, morbidity, mortality, conversion, anastomotic leak, surgical site infection and re-operation.

The MeSH terms related to the colorectal cancer, SILS and MILS were identified from the PubMed and subsequently inserted in the search boxes of other electronic databases. Attempts to find extra trials were also made by the hand searching of the references of published studies.

\section{Data management}

Three reviewers (MSS, MH and WR) independently searched and selected studies and disagreements were resolved by consensus. Inclusion criteria were as follows: RCT in patients with colorectal disorders needing surgical resection; surgical resection was done by SILS versus MILS approach; and had at least one outcomes of interest as described above. The studies were included regardless of sample size, origin of study, age of the participant, gender of the participant and the language in which the study was published.

\section{Interventions}

Our interventions of interest were SILS versus MILS for colorectal resection for any reason.

\section{Outcome of interest}

We studied outcomes such as duration of operation, length of stay in hospital, lymph nodes harvesting, morbidity, mortality, conversion rate, anastomotic leak, surgical site infection and re-operation. These outcomes were defined according to the original studies.

\section{Risk of bias assessment}

The methodological quality of the included studies was assessed by MSS and MH as recommended by the Cochrane 


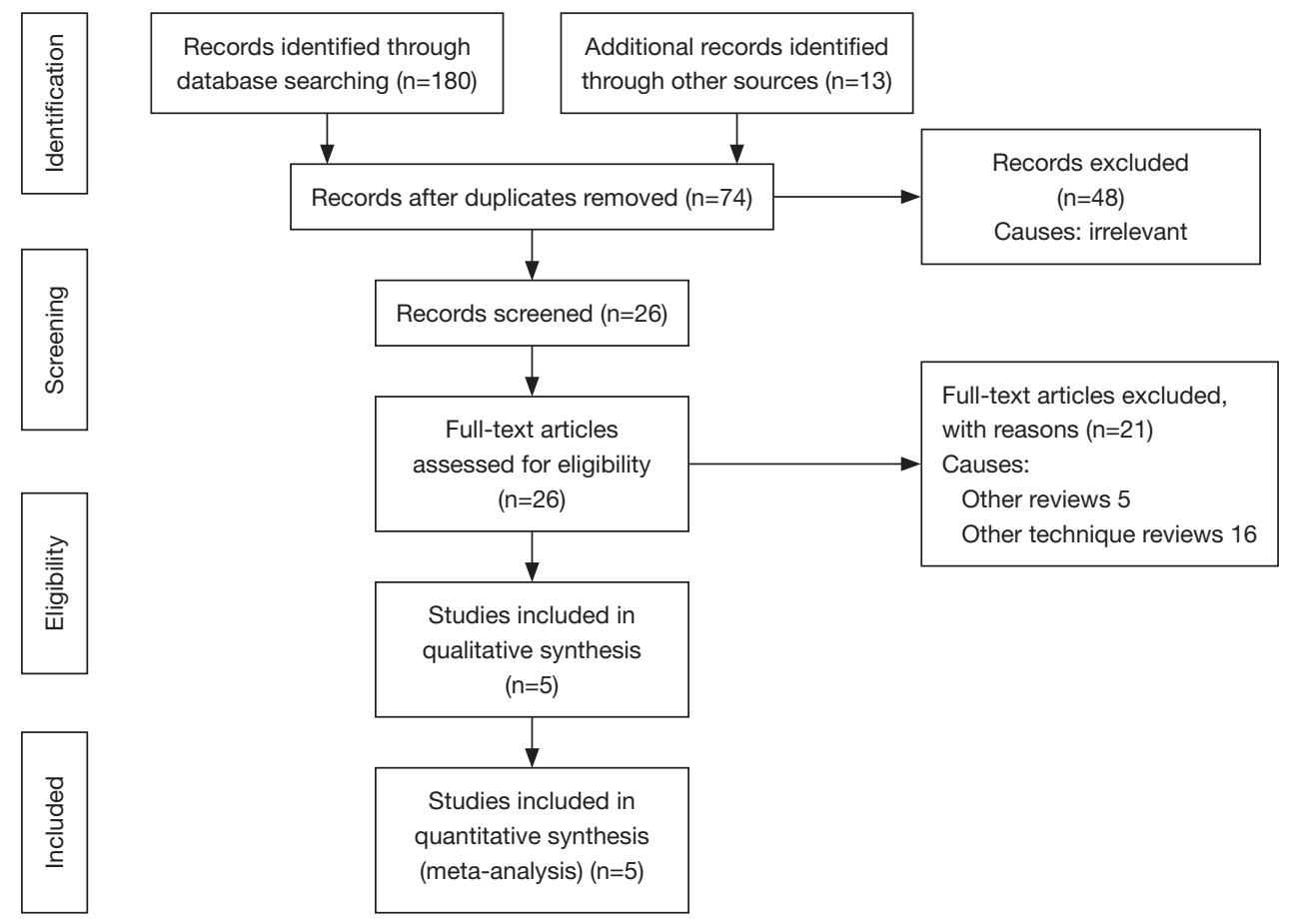

Figure 1 PRISMA flow diagram.

Collaboration using the "Risk of Bias Assessment Tool". This included random sequence generation, allocation concealment, blinding of participants and personnel, blinding of outcome assessment, incomplete outcome data, and selective outcome reporting. Disagreement was resolved by a third reviewer (WR). The critical appraisal tool to score the quality of included trials was also adopted from the published guidelines of Jadad et al. (43) and Chalmers et al. (44). The short summary of the resulting evidence was presented in a tabulated form by using tool GradePro ${ }^{\circledR}(45)$, provided by the Cochrane Collaboration.

\section{Statistical analysis}

The efficacy of SILS versus MILS was directly compared and pooled for each outcome of interest if there were at least two studies for each comparison. The risk ratio (RR) was estimated and pooled across studies using a randomeffect model. Heterogeneity was assessed using Cochrane $\mathrm{Q}$ test and $\mathrm{I}^{2}$ statistic. The statistical analysis of the data was conducted according to the guidelines provided by the Cochrane Collaboration including the use of RevMan $5.3{ }^{\circledR}$ statistical software, and the use of forest plots for the graphical display of the combined outcomes (46-52).

\section{Results}

\section{Characteristics of selected trials:}

A total of 194 studies were identified from Scopus and Medline and other electronic databases. Among them, 5 RCTs ( $\mathrm{n}=525$ ), published between 2012 and 2018, were eligible for inclusion $(17,22,37,53,54)$; and the reasons for ineligibility are described in PRISMA flow chart Figure 1. The salient characteristics of included trials are described in Table 1. Table 2 highlights the operative technique used in both approaches. All 5 RCTs had two intervention arms, i.e., SILS versus MILS (N=265, $\mathrm{n}=260)$. The extracted data from the included trials is given in Table 3 and this data was used to achieve the summated outcome for overall effect size.

\section{Risk of bias in selected studies}

The reported quality variables in included trials are given in Table 4. Summary of the evidence analysed in the GRADE-Pro tool is given in Figure 2 denoting the high quality of evidence based upon outcomes as per protocol scoring systems. Among 5 studies, $90 \%$ were considered at low risk of bias due to the presence of random sequence generation, allocation concealment (selection bias), blinding of participants, blinding 
Table 1 Characteristics of included randomized controlled trials

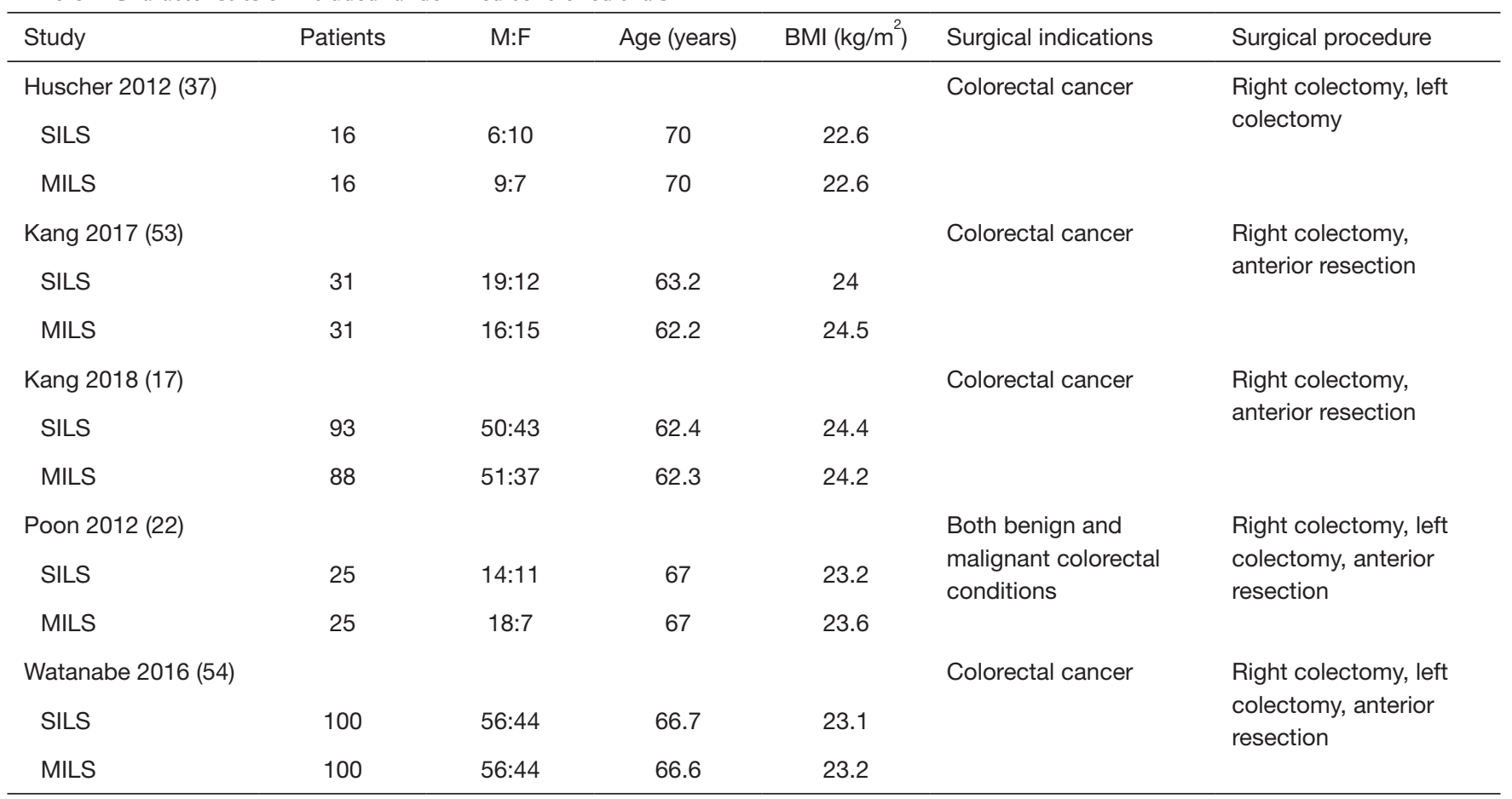

SILS, single incision laparoscopic surgery; MILS, multi-incision laparoscopic surgery.

Table 2 Surgical techniques used in both groups

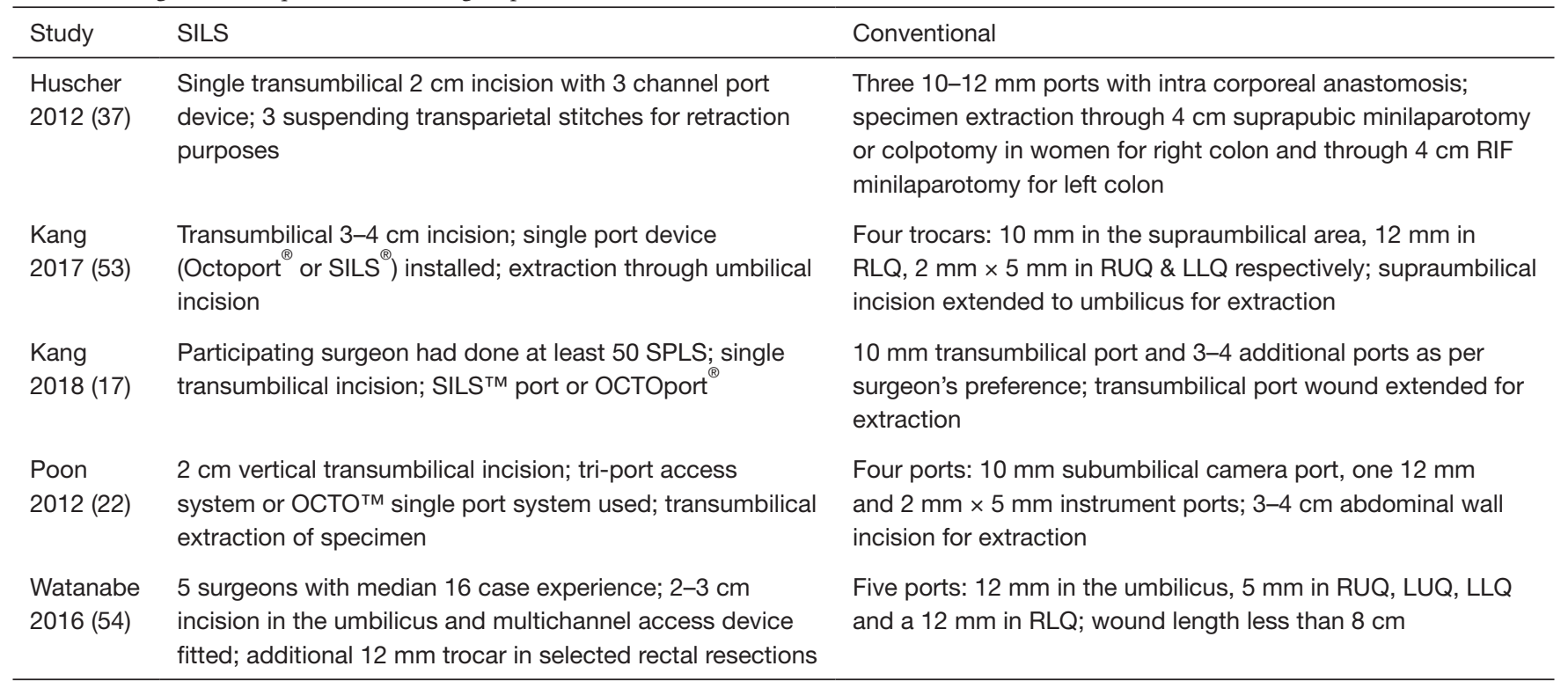


Table 3 The extracted data used for meta-analysis

\begin{tabular}{|c|c|c|c|c|c|c|c|c|c|}
\hline Study & $\begin{array}{l}\text { Operation time } \\
\text { (minutes) }\end{array}$ & $\begin{array}{c}\text { Conversion, } \\
\mathrm{n}\end{array}$ & $\begin{array}{l}\text { Stay } \\
\text { (days) }\end{array}$ & $\begin{array}{c}\text { Mortality, } \\
\mathrm{n}\end{array}$ & $\begin{array}{c}\text { Complications, } \\
\mathrm{n}\end{array}$ & $\begin{array}{l}\text { Leak/abd } \\
\text { abscess, n }\end{array}$ & SSI, n & $\begin{array}{c}\text { Re-operation, } \\
\mathrm{n}\end{array}$ & $\begin{array}{c}\text { Lymph nodes, } \\
\mathrm{n}\end{array}$ \\
\hline \multicolumn{10}{|c|}{ Huscher 2012 (37) } \\
\hline MILS & $129 \pm 46$ & $0 / 16$ & $7 \pm 2$ & $0 / 16$ & $5 / 16$ & $1 / 16$ & $2 / 16$ & $1 / 16$ & $16 \pm 5$ \\
\hline MILS & $130.9 \pm 34.2$ & $0 / 31$ & $6.5 \pm 1.4$ & $0 / 31$ & $4 / 31$ & $0 / 31$ & $1 / 31$ & $0 / 31$ & $20.1 \pm 8.9$ \\
\hline \multicolumn{10}{|c|}{ Kang 2018 (17) } \\
\hline SILS & $189.4 \pm 80.5^{\star}$ & $14 / 93$ & $7 \pm 4.75$ & $0 / 93$ & $18 / 94$ & $0 / 93$ & $4 / 93$ & $1 / 93$ & $25 \pm 12^{*}$ \\
\hline MILS & $124 \pm 36.5$ & $0 / 25$ & $5 \pm 1$ & $0 / 25$ & $3 / 25$ & $0 / 25$ & $2 / 25$ & $0 / 25$ & $20 \pm 8$ \\
\hline \multicolumn{10}{|c|}{ Watanabe 2016 (54) } \\
\hline SILS & $156 \pm 37$ & $1 / 100$ & $6 \pm 0.25^{\star}$ & $0 / 100$ & $12 / 100$ & $2 / 100$ & $0 / 100$ & $3 / 100$ & $25.5 \pm 11.4$ \\
\hline MILS & $162 \pm 36$ & $2 / 100$ & $6 \pm 0.25^{*}$ & $0 / 100$ & $15 / 100$ & $4 / 100$ & $3 / 100$ & $3 / 100$ & $24.1 \pm 11$ \\
\hline
\end{tabular}

*, the standard deviation was either estimated from range value or P value. SILS, single incision laparoscopic surgery; MILS, multi-incision laparoscopic surgery

Table 4 The relevant quality indicators reported in the included trials

\begin{tabular}{|c|c|c|c|c|c|c|}
\hline Study & Randomization technique & Blinding & Concealment & Intention to treat analysis & Trial registration & Ethics approval \\
\hline Huscher 2012 (37) & $\begin{array}{l}\text { Controlled stratified } \\
\text { randomisation }\end{array}$ & Yes & Yes & Yes & Not reported & Not reported \\
\hline Kang 2018 (17) & Web based registration & No & Yes & Yes & ClinicalTrials.gov & Yes \\
\hline Watanabe 2016 (54) & $\begin{array}{l}\text { Computerised } \\
\text { randomisation }\end{array}$ & No & No & Yes & $\begin{array}{l}\text { Japanese Clinical } \\
\text { Trials Registry }\end{array}$ & Yes \\
\hline
\end{tabular}

of outcome assessment (performance bias), data management (attribution bias), incomplete outcome data (detection bias) and selective outcome reporting (reporting bias).

\section{Operation time for SILS versus MILS}

There was significant heterogeneity $\left[\mathrm{Tau}^{2}=0.07, \mathrm{chi}^{2}\right.$ $\left.=10.77, \mathrm{df}=4(\mathrm{P}=0.03) ; \mathrm{I}^{2}=63 \%\right]$ among included trials.
Therefore, in the random effects model (SMD, 0.20; 95\% CI, -0.11 to $0.52 ; \mathrm{z}=1.28 ; \mathrm{P}=0.20$; Figure 3 ) analysis, the duration of operation was not influenced by the surgical approach for colorectal resections.

\section{Duration of hospitalization following SILS versus MILS}

There was significant heterogeneity $\left[\mathrm{Tau}^{2}=0.08, \mathrm{chi}^{2}\right.$ 


\begin{tabular}{|c|c|c|c|c|c|c|}
\hline \multicolumn{7}{|c|}{ Single incision laparoscopic surgery compared to multi-incision laparoscopic surgery for colorectal resections } \\
\hline \multicolumn{7}{|c|}{$\begin{array}{l}\text { Patient or population: patients with colorectal resections } \\
\text { Settings: } \\
\text { Intervention: Single incision laparoscopic surgery } \\
\text { Comparison: Multi-incision laparoscopic surgery }\end{array}$} \\
\hline \multirow[t]{2}{*}{ Outcomes } & \multicolumn{2}{|c|}{ Illustrative comparative risks $(95 \% \mathrm{Cl})$} & \multirow{2}{*}{$\begin{array}{l}\text { Relative effect } \\
(95 \% \mathrm{Cl})\end{array}$} & \multirow{2}{*}{$\begin{array}{l}\text { No. of } \\
\text { participants } \\
\text { (studies) }\end{array}$} & \multirow{2}{*}{$\begin{array}{l}\text { Quality } \\
\text { of the } \\
\text { evidence } \\
\text { (GRADE) }\end{array}$} & \multirow{2}{*}{ Comments } \\
\hline & $\begin{array}{l}\text { Assumed risk } \\
\text { Multi-incision } \\
\text { laparoscopic surgery }\end{array}$ & $\begin{array}{l}\text { Corresponding risk } \\
\text { Single incision laparoscopic surgery }\end{array}$ & & & & \\
\hline $\begin{array}{l}\text { Operation time } \\
\text { Standardized mean } \\
\text { difference } \\
\text { Follow-up: mean } 8 \text { weeks }\end{array}$ & & $\begin{array}{l}\text { The mean operation time in the } \\
\text { intervention groups was } 0.2 \text { standard } \\
\text { deviations higher }(0.11 \text { lower to } 0.52 \\
\text { higher) }\end{array}$ & & $\begin{array}{l}525 \\
\text { (5 studies) }\end{array}$ & $\begin{array}{l}++++ \\
\text { high }\end{array}$ & $\begin{array}{l}\text { SMD } 0.2 \\
(-0.11 \text { to } 0.52)\end{array}$ \\
\hline $\begin{array}{l}\text { Length of stay } \\
\text { Standardized mean } \\
\text { difference } \\
\text { Follow-up: mean } 8 \text { weeks }\end{array}$ & & $\begin{array}{l}\text { The mean length of stay in the } \\
\text { intervention groups was } 0.18 \text { standard } \\
\text { deviations lower ( } 0.51 \text { lower to } 0.14 \\
\text { higher) }\end{array}$ & & $\begin{array}{l}525 \\
\text { (5 studies) }\end{array}$ & $\begin{array}{l}++++ \\
\text { high }\end{array}$ & $\begin{array}{l}\text { SMD }-0.18 \\
(-0.51 \text { to } 0.14)\end{array}$ \\
\hline $\begin{array}{l}\text { Lymph node harvesting } \\
\text { Standardized mean } \\
\text { difference } \\
\text { Follow-up: mean } 8 \text { weeks }\end{array}$ & & $\begin{array}{l}\text { The mean lymph node harvesting in the } \\
\text { intervention groups was } 0.09 \text { standard } \\
\text { deviations higher ( } 0.14 \text { lower to } 0.33 \\
\text { higher) }\end{array}$ & & $\begin{array}{l}525 \\
\text { (5 studies) }\end{array}$ & $\begin{array}{l}++++ \\
\text { high }\end{array}$ & $\begin{array}{l}\text { SMD } 0.09 \\
(-0.14 \text { to } 0.33)\end{array}$ \\
\hline \multirow{4}{*}{$\begin{array}{l}\text { Complications } \\
\text { Risk ratio } \\
\text { Follow-up: mean } 8 \text { weeks }\end{array}$} & Study population & & \multirow{4}{*}{$\begin{array}{l}\text { RR } 1 \\
(0.65 \text { to } 1.54)\end{array}$} & \multirow{4}{*}{$\begin{array}{l}525 \\
\text { (5 studies) }\end{array}$} & \multirow{4}{*}{$\begin{array}{l}++++ \\
\text { high }\end{array}$} & \\
\hline & 162 per 1,000 & 162 per 1,000 (105 to 249$)$ & & & & \\
\hline & Moderate & & & & & \\
\hline & 150 per 1,000 & 150 per 1,000 (97 to 231$)$ & & & & \\
\hline \multirow{4}{*}{$\begin{array}{l}\text { Mortality } \\
\text { Risk ratio } \\
\text { Follow-up: mean } 8 \text { weeks }\end{array}$} & Study population & & \multirow{4}{*}{$\begin{array}{l}\text { RR } 3 \\
(0.13 \text { to } 70.92)\end{array}$} & \multirow{4}{*}{$\begin{array}{l}525 \\
\text { (5 studies) }\end{array}$} & \multirow{4}{*}{$\begin{array}{l}++++ \\
\text { high }^{++}\end{array}$} & \\
\hline & 0 per 1,000 & 0 per 1,000 (0 to 0$)$ & & & & \\
\hline & Moderate & & & & & \\
\hline & 0 per 1,000 & 0 per $1,000(0$ to 0$)$ & & & & \\
\hline \multirow{4}{*}{$\begin{array}{l}\text { Conversion } \\
\text { Risk ratio } \\
\text { Follow-up: mean } 8 \text { weeks }\end{array}$} & Study population & & \multirow{4}{*}{$\begin{array}{l}\mathrm{RR} 4.43 \\
(0.63 \text { to } 31.17)\end{array}$} & \multirow{4}{*}{$\begin{array}{l}525 \\
\text { (5 studies) }\end{array}$} & \multirow{4}{*}{$\begin{array}{l}++++ \\
\text { high }\end{array}$} & \\
\hline & 8 per 1,000 & 34 per 1,000 (5 to 240$)$ & & & & \\
\hline & Moderate & & & & & \\
\hline & 0 per 1,000 & 0 per $1,000(0$ to 0$)$ & & & & \\
\hline \multirow{4}{*}{$\begin{array}{l}\text { Anastomotic leak } \\
\text { Risk ratio } \\
\text { Follow-up: mean } 8 \text { weeks }\end{array}$} & Study population & & \multirow{4}{*}{$\begin{array}{l}\text { RR } 0.43 \\
(0.11 \text { to } 1.63)\end{array}$} & \multirow{4}{*}{$\begin{array}{l}525 \\
\text { (5 studies) }\end{array}$} & \multirow{4}{*}{$\begin{array}{l}++++ \\
\text { high }\end{array}$} & \\
\hline & 23 per 1,000 & 10 per 1,000 (3 to 38$)$ & & & & \\
\hline & Moderate & & & & & \\
\hline & 11 per 1,000 & 5 per 1,000 (1 to 18$)$ & & & & \\
\hline \multirow{4}{*}{$\begin{array}{l}\text { Surgical site infection } \\
\text { Risk ratio } \\
\text { Follow-up: mean } 8 \text { weeks }\end{array}$} & Study population & & \multirow{4}{*}{$\begin{array}{l}\text { RR } 0.58 \\
(0.23 \text { to } 1.48)\end{array}$} & \multirow{4}{*}{$\begin{array}{l}525 \\
\text { (5 studies) }\end{array}$} & \multirow{4}{*}{$\begin{array}{l}++++ \\
\text { high }\end{array}$} & \\
\hline & 46 per 1,000 & 27 per 1,000 (11 to 68$)$ & & & & \\
\hline & Moderate & & & & & \\
\hline & 46 per 1,000 & 27 per 1,000 (11 to 68$)$ & & & & \\
\hline \multirow{4}{*}{$\begin{array}{l}\text { Re-operation } \\
\text { Risk ratio } \\
\text { Follow-up: mean } 8 \text { weeks }\end{array}$} & Study population & & \multirow{4}{*}{$\begin{array}{l}\text { RR } 0.62 \\
\text { (0.19 to } 2.03)\end{array}$} & 525 & ++++ & \\
\hline & 27 per 1,000 & 17 per 1,000 (5 to 55$)$ & & & & \\
\hline & Moderate & & & & & \\
\hline & 30 per 1,000 & 19 per $1,000(6$ to 61$)$ & & & & \\
\hline
\end{tabular}

The basis for the assumed risk (e.g., the median control group risk across studies) is provided in footnotes. The corresponding risk (and its $95 \%$ confidence interval) is based on the assumed risk in the comparison group and the relative effect of the intervention (and its $95 \% \mathrm{Cl}$ )

$\mathrm{Cl}$ : confidence interval, RR: risk ratio;

\section{GRADE Working Group grades of evidence}

High quality: Further research is very unlikely to change our confidence in the estimate of effect

Moderate quality: Further research is likely to have an important impact on our confidence in the estimate of effect and may change the estimate

Low quality: Further research is very likely to have an important impact on our confidence in the estimate of effect and is likely to change the estimate Very low quality: We are very uncertain about the estimate

"No explanation was provided

223 Figure 2 GRADE-Pro summary of evidence. 


\begin{tabular}{|c|c|c|c|c|c|c|c|c|c|}
\hline \multirow[b]{2}{*}{ Study or Subgroup } & \multicolumn{3}{|c|}{ SILS } & \multicolumn{3}{|c|}{ MILS } & \multicolumn{2}{|c|}{ Std. Mean Difference } & \multirow{2}{*}{$\begin{array}{c}\text { Std. Mean Difference } \\
\text { IV, Random, } 95 \% \mathrm{Cl}\end{array}$} \\
\hline & Mean & SD & Total & Mean & SD & Total & Weight & IV, Random, 95\% Cl & \\
\hline Huscher 2012 & 146 & 61 & 16 & 129 & 46 & 16 & $12.6 \%$ & $0.31[-0.39,1.00]$ & \\
\hline Kang 2017 & 134.9 & 48.3 & 31 & 130.9 & 34.2 & 31 & $18.3 \%$ & $0.09[-0.40,0.59]$ & \\
\hline Poon 2012 & 155 & 36 & 25 & 124 & 36.5 & 25 & $15.7 \%$ & $0.84[0.26,1.42]$ & \\
\hline Watanabe 2016 & 156 & 37 & 100 & 162 & 36 & 100 & $27.0 \%$ & $-0.16[-0.44,0.11]$ & \\
\hline \multicolumn{9}{|c|}{$\begin{array}{l}\text { Heterogeneity: } \text { Tau }^{2}=0.07 ; \mathrm{Chi}^{2}=10.77, \mathrm{df}=4(\mathrm{P}=0.03) ; \mathrm{I}^{2}=63 \% \\
\text { Test for overall effect: } Z=1.28(P=0.20)\end{array}$} & $\begin{array}{cccc} & 1 & 1 & 1 \\
-1 & -0.5 & 0 & 0.5 \\
\text { Favours SILS } & \text { Favours I }\end{array}$ \\
\hline
\end{tabular}

Figure 3 Forest plot for duration of operation following use of single incision laparoscopic surgery versus conventional multi-incision laparoscopic surgery for colorectal resections. Standardized mean difference is shown with 95 per cent confidence intervals.

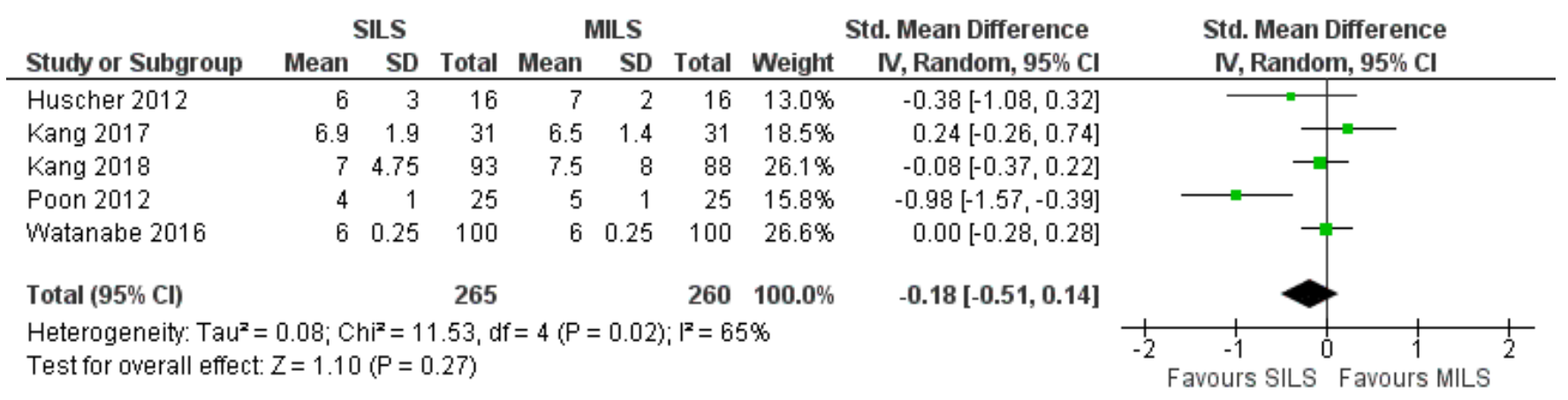

Figure 4 Forest plot for duration of hospital stay following use of single incision laparoscopic surgery versus conventional multi-incision laparoscopic surgery for colorectal resections. Standardized mean difference is shown with 95 per cent confidence intervals.

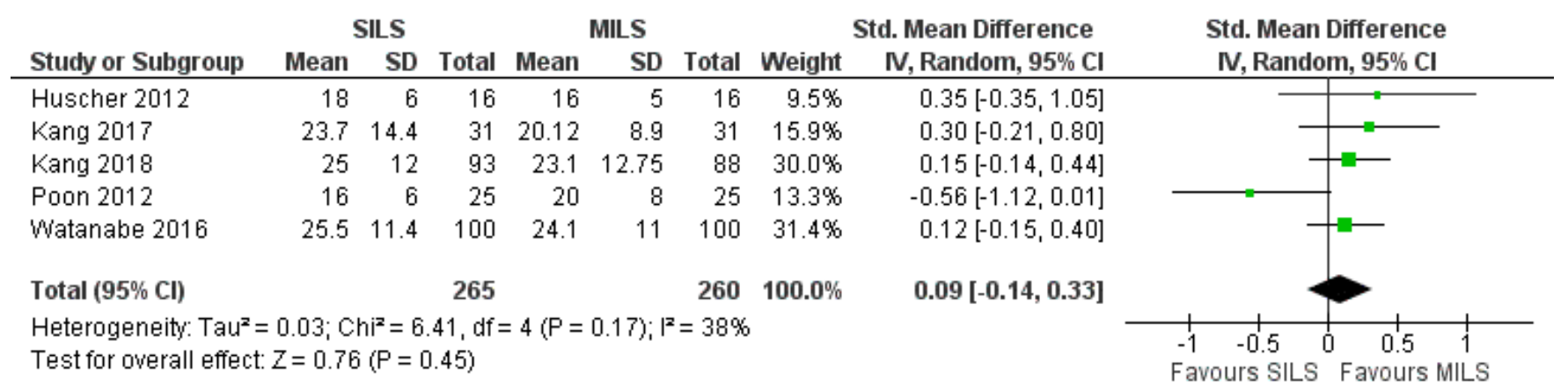

Figure 5 Forest plot for lymph node harvesting following use of single incision laparoscopic surgery versus conventional multi-incision laparoscopic surgery for colorectal resections. Standardized mean difference is shown with 95 per cent confidence intervals.

$=11.53, \mathrm{df}=4(\mathrm{P}=0.02) ; \mathrm{I}^{2}=65 \%$ ] among trials. Therefore, in the random effects model (SMD, -0.18 ; 95\% CI, -0.51 to $0.14 ; \mathrm{z}=1.10 ; \mathrm{P}=0.27$; Figure 4 ) analysis, the tenure of hospitalization was statistically similar following the use of any approach for colorectal resection.

(C) Translational Gastroenterology and Hepatology. All rights reserved.

\section{Lymph node harvesting difference for oncological safety}

There was no heterogeneity $\left[\mathrm{Tau}^{2}=0.03 \mathrm{chi}^{2}=6.41, \mathrm{df}\right.$ $\left.=4(\mathrm{P}=0.17) ; \mathrm{I}^{2}=38 \%\right]$ among trials. In the random effects model (SMD, 0.09; 95\% CI, -0.14 to $0.33 ; \mathrm{z}=0.76 ; \mathrm{P}=0.45$; Figure 5) analysis, both procedures offer similar oncological 


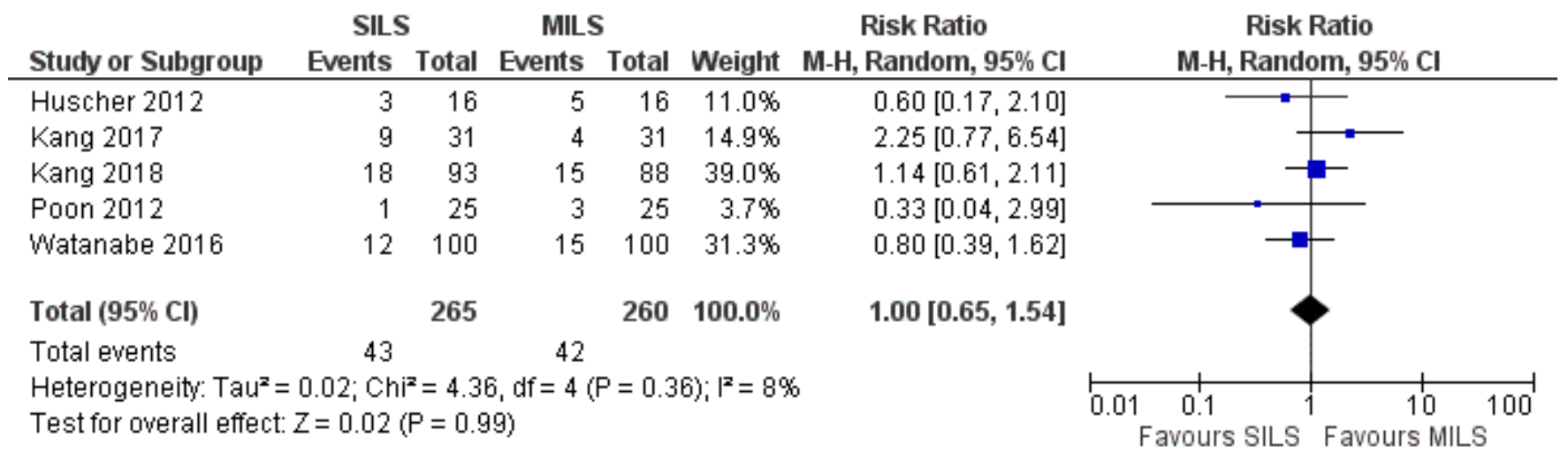

Figure 6 Forest plot for post-operative complications following use of single incision laparoscopic surgery versus conventional multiincision laparoscopic surgery for colorectal resections. Risk ratios are shown with 95 per cent confidence intervals.

\begin{tabular}{|c|c|c|c|c|c|c|c|c|}
\hline Study or Subgroup & $\begin{array}{l}\text { SILS } \\
\text { Events }\end{array}$ & Total & \multicolumn{2}{|c|}{ MILS } & Weight & $\begin{array}{c}\text { Risk Ratio } \\
\text { M-H, Random, } 95 \% \mathrm{Cl}\end{array}$ & \multicolumn{2}{|c|}{$\begin{array}{c}\text { Risk Ratio } \\
\text { M-H, Random, } 95 \% \mathrm{Cl}\end{array}$} \\
\hline Huscher 2012 & 0 & 16 & 0 & 16 & & Not estimable & & \\
\hline Kang 2017 & 1 & 31 & 0 & 31 & $100.0 \%$ & $3.00[0.13,70.92]$ & & \\
\hline Kang 2018 & 0 & 93 & 0 & 88 & & Not estimable & & \\
\hline Poon 2012 & 0 & 25 & 0 & 25 & & Not estimable & & \\
\hline Watanabe 2016 & 0 & 100 & 0 & 100 & & Not estimable & & \\
\hline Total $(95 \% \mathrm{Cl})$ & & 265 & & 260 & $100.0 \%$ & $3.00[0.13,70.92]$ & & \\
\hline Total events & 1 & & 0 & & & & & \\
\hline $\begin{array}{l}\text { Heterogeneity: Not a } \\
\text { Test for overall effect }\end{array}$ & $\begin{array}{l}\text { plicable } \\
Z=0.68\end{array}$ & $P=0.5$ & & & & & $\begin{array}{|cc|}0.01 & 0.1 \\
& \\
& \text { Favours SILS }\end{array}$ & $\left.\right|_{\text {Favours MILS }} ^{10} 100$ \\
\hline
\end{tabular}

Figure 7 Forest plot for mortality following use of single incision laparoscopic surgery versus conventional multi-incision laparoscopic surgery for colorectal resections. Risk ratios are shown with 95 per cent confidence intervals.

safety in terms of total number of lymph nodes removed at the time of radical resection for colorectal cancer.

\section{Peri-procedural/post-procedural morbidity and mortality}

There was no heterogeneity $\left[\mathrm{Tau}^{2}=0.02, \mathrm{chi}^{2}=4.36, \mathrm{df}=4\right.$ $\left.(\mathrm{P}=0.36) ; \mathrm{I}^{2}=8 \%\right]$ among included trials. In the random effects model (RR, 1.0; 95\% CI, 0.65-1.54; $\mathrm{z}=0.02 ; \mathrm{P}=0.99$; Figure 6) analysis, the procedural morbidity and mortality (RR, 3.0; 95\% CI, 0.13-70.92; z=0.68; $\mathrm{P}=0.50$; Figure 7) were also not different in both groups.

\section{Risk of conversion to open procedure}

There was no heterogeneity $\left[\mathrm{Tau}^{2}=1.96, \mathrm{chi}^{2}=5.95, \mathrm{df}=3\right.$ $\left.(\mathrm{P}=0.11) ; \mathrm{I}^{2}=50 \%\right]$ among included studies. In the random effects model (RR, 4.43; 95\% CI, 0.63-31.17; $\mathrm{z}=1.50$; $\mathrm{P}=0.13$; Figure 8 ) analysis, the conversion rate for SILS and MILS was similar.

\section{Colorectal anastomotic leak}

There was no heterogeneity $\left[\mathrm{Tau}^{2}=0.00, \mathrm{chi}^{2}=0.09, \mathrm{df}=2\right.$ $\left.(\mathrm{P}=0.95) ; \mathrm{I}^{2}=0 \%\right]$ among included studies. In the random effects model (RR, 0.43; 95\% CI, 0.11-1.63; $\mathrm{z}=1.24 ; \mathrm{P}=0.21$; Figure 9) analysis, the colorectal anastomotic leak risk was not influenced by the surgical approach for colorectal resection.

\section{Influence on the surgical site infection and re-operation rate}

There was no heterogeneity $\left[\mathrm{Tau}^{2}=0.00, \mathrm{chi}^{2}=1.56, \mathrm{df}=4\right.$ $\left.(\mathrm{P}=0.82) ; \mathrm{I}^{2}=0 \%\right]$ among included studies. In the random 


\begin{tabular}{|c|c|c|c|c|c|c|c|c|}
\hline \multirow[b]{2}{*}{ Study or Subgroup } & \multicolumn{2}{|c|}{ SILS } & \multicolumn{2}{|c|}{ MILS } & \multirow[b]{2}{*}{ Weight } & \multirow{2}{*}{$\begin{array}{c}\text { Risk Ratio } \\
\text { M-H, Random, } 95 \% \mathrm{Cl}\end{array}$} & \multirow{2}{*}{\multicolumn{2}{|c|}{$\begin{array}{c}\text { Risk Ratio } \\
\text { M-H, Random, } 95 \% \mathrm{Cl}\end{array}$}} \\
\hline & Events & Total & Events & Total & & & & \\
\hline Huscher 2012 & 1 & 16 & 0 & 16 & $22.0 \%$ & $3.00[0.13,68.57]$ & & $\longrightarrow$ \\
\hline Kang 2017 & 6 & 31 & 0 & 31 & $24.5 \%$ & $13.00[0.76,221.26]$ & & $\longrightarrow$ \\
\hline Kang 2018 & 14 & 93 & 0 & 88 & $24.7 \%$ & $27.46[1.66,453.45]$ & & $\longrightarrow$ \\
\hline Poon 2012 & 0 & 25 & 0 & 25 & & Not estimable & & \\
\hline Watanabe 2016 & 1 & 100 & 2 & 100 & $28.8 \%$ & $0.50[0.05,5.43]$ & & \\
\hline Total (95\% Cl) & & 265 & & 260 & $100.0 \%$ & $4.43[0.63,31.17]$ & & \\
\hline Total events & 22 & & 2 & & & & & \\
\hline $\begin{array}{l}\text { Heterogeneity: Tau } \\
\text { Test for overall effe }\end{array}$ & $\begin{array}{l}1.96 ; \mathrm{Ch} \\
\mathrm{z}=1.50\end{array}$ & $\begin{array}{l}P=5.9 \\
P=0 .\end{array}$ & $\begin{array}{l}5, d f=3 \\
\text { 3) }\end{array}$ & $P=0.1$ & 1); $\left.\right|^{2}=50$ & & $\begin{array}{cc}0.01 & 0.1 \\
& \\
\text { Favours SILS } & 1\end{array}$ & $\begin{array}{c}10 \\
\text { Favours MILS }\end{array}$ \\
\hline
\end{tabular}

Figure 8 Forest plot for conversion following use of single incision laparoscopic surgery versus conventional multi-incision laparoscopic surgery for colorectal resections. Risk ratios are shown with 95 per cent confidence intervals.

\begin{tabular}{|c|c|c|c|c|c|c|c|c|}
\hline Study or Subgroup & \multicolumn{2}{|c|}{ SILS } & \multicolumn{2}{|c|}{ MILS } & Weight & $\begin{array}{c}\text { Risk Ratio } \\
\text { M-H, Random, } 95 \% \mathrm{Cl}\end{array}$ & \multicolumn{2}{|c|}{$\begin{array}{c}\text { Risk Ratio } \\
\text { M-H, Random, } 95 \% \mathrm{Cl}\end{array}$} \\
\hline Huscher 2012 & 0 & 16 & 1 & 16 & $18.3 \%$ & $0.33[0.01,7.62]$ & & \\
\hline Kang 2017 & 0 & 31 & 0 & 31 & & Not estimable & & \\
\hline Kang 2018 & 0 & 93 & 1 & 88 & $17.7 \%$ & $0.32[0.01,7.65]$ & & \\
\hline Poon 2012 & 0 & 25 & 0 & 25 & & Not estimable & & \\
\hline Watanabe 2016 & 2 & 100 & 4 & 100 & $64.0 \%$ & $0.50[0.09,2.67]$ & & \\
\hline Total $(95 \% \mathrm{Cl})$ & & 265 & & 260 & $100.0 \%$ & $0.43[0.11,1.63]$ & & \\
\hline Total events & 2 & & 6 & & & & & \\
\hline $\begin{array}{l}\text { Heterogeneity: Tau } \\
\text { Test for overall effect }\end{array}$ & $\begin{array}{l}0.00 ; \mathrm{Chi} \\
Z=1.24\end{array}$ & $\begin{array}{l}P=0.09 \\
P=0.2\end{array}$ & $\begin{array}{l}9, d f=2( \\
1)\end{array}$ & $=0.9$ & $15) ;\left.\right|^{2}=0 \%$ & & $\begin{array}{|cc|}0.01 & 0.1 \\
\text { Favours SILS }\end{array}$ & \begin{tabular}{|c|}
10 \\
Favours MILS \\
\end{tabular} \\
\hline
\end{tabular}

Figure 9 Forest plot for anastomotic leak following use of single incision laparoscopic surgery versus conventional multi-incision laparoscopic surgery for colorectal resections. Risk ratios are shown with 95 per cent confidence intervals.

effects model (RR, 0.58; 95\% CI, 0.23-1.48; z=1.15; $\mathrm{P}=0.25$; Figure 10) analysis, the surgical site infection rate and re-operation rate (RR, 0.62; 95\% CI, 0.19-2.03; $\mathrm{z}=0.80$; $\mathrm{P}=0.43$; Figure 11) were also not influenced by the surgical approach for colorectal resection.

\section{Discussion}

\section{Summary of main results}

The major findings of this review article highlight the clinical, oncological and technical effectiveness of SILS comparing with the conventional MILS for colorectal resections. Findings of current meta-analysis of five RCTs on 525 patients reiterate that the colorectal resections following SILS versus MILS technique are similar in terms of operation time, length of in-patient stay and lymph node harvesting. Furthermore, post-operative morbidity, postoperative mortality, surgical site infection rate, anastomotic leak rate, conversion rate and re-operation rate were also statistically similar following SILS and MILS. Therefore, SILS failed to demonstrate any superiority over MILS for colorectal resections in all post-operative surgical outcomes.

\section{Application and completeness of evidence in this study}

The inclusion criterion for this study was strict and was confined to the combined evaluation of published RCTs only. The findings of current study are appropriate and applicable to colorectal patients who require right hemicolectomy, left hemicolectomy and anterior resection only. The feasibility of SILS for other colorectal procedures still needs evaluation before making final conclusion. 


\begin{tabular}{|c|c|c|c|c|c|c|c|c|}
\hline \multirow[b]{2}{*}{ Study or Subgroup } & \multicolumn{2}{|c|}{ SILS } & \multicolumn{2}{|c|}{ MILS } & \multirow[b]{2}{*}{ Weight } & Risk Ratio & \multirow{2}{*}{\multicolumn{2}{|c|}{\begin{tabular}{c}
\multicolumn{1}{c}{ Risk Ratio } \\
M-H, Random, $95 \% \mathrm{Cl}$
\end{tabular}}} \\
\hline & Events & Total & Events & Total & & M-H, Random, $95 \% \mathrm{Cl}$ & & \\
\hline Huscher 2012 & 1 & 16 & 2 & 16 & $16.7 \%$ & $0.50[0.05,4.98]$ & & \\
\hline Kang 2017 & 0 & 31 & 1 & 31 & $8.8 \%$ & $0.33[0.01,7.88]$ & & \\
\hline Kang 2018 & 4 & 93 & 4 & 88 & $48.1 \%$ & $0.95[0.24,3.67]$ & & \\
\hline Poon 2012 & 1 & 25 & 2 & 25 & $16.2 \%$ & $0.50[0.05,5.17]$ & & \\
\hline Watanabe 2016 & 0 & 100 & 3 & 100 & $10.1 \%$ & $0.14[0.01,2.73]$ & & \\
\hline Total (95\% Cl) & & 265 & & 260 & $100.0 \%$ & $0.58[0.23,1.48]$ & & \\
\hline Total events & 6 & & 12 & & & & & \\
\hline $\begin{array}{l}\text { Heterogeneity: } \mathrm{Tau}^{2} \\
\text { Test for overall effect }\end{array}$ & $\begin{array}{l}0.00 ; \text { Chi } \\
Z=1.15\end{array}$ & $\begin{array}{l}z=1.56 \\
P=0.2\end{array}$ & $\begin{array}{l}6, d f=4(1 \\
5)\end{array}$ & $P=0.8$ & $2) ; I^{2}=0 \%$ & & $\begin{array}{c}0.010 .1 \\
\text { Favours SILS }\end{array}$ & ${ }^{10} 100$ \\
\hline
\end{tabular}

Figure 10 Forest plot for surgical site infection following use of single incision laparoscopic surgery versus conventional multi-incision laparoscopic surgery for colorectal resections. Risk ratios are shown with 95 per cent confidence intervals.

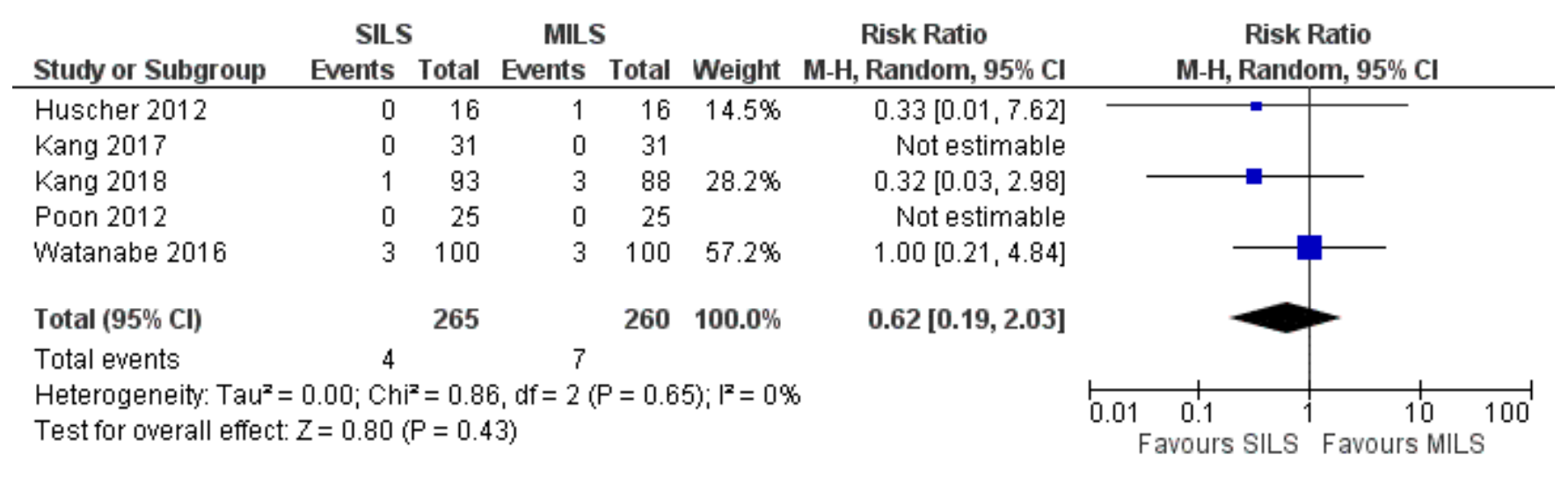

Figure 11 Forest plot for re-operation rate following use of single incision laparoscopic surgery versus conventional multi-incision laparoscopic surgery for colorectal resections. Risk ratios are shown with 95 per cent confidence intervals.

\section{Strength of the presented evidence}

Authors have used standard quality assessment tools to conduct this study. The evaluation of the included trials was performed using multi-pronged approach such as risk of bias assessment tool by the Cochrane Collaboration, Jadad and Chalmers et al. scoring tools; and presentation of summary of evidence from GRADE-pro tool. Overall strength of evidence may be considered high as depicted in Figure 2. Diverse inclusion and exclusion criteria, variable experience of operating surgeon and evaluation of simple colorectal resection by MILS approach may also be a source of biased outcome in RCTs.

\section{Potential biases in the review process}

The variable experience of operating surgeon, study of different colorectal resections, exclusion of more complex colorectal resections such as subtotal colectomy, segmental resection and abdominoperineal resection might have influenced the outcomes. Other confounding factors which might have influenced the final outcome may be the use of different operating tools, variable size of umbilical incision and variable number of ports in MILS technique.

\section{Agreement and disagreement with other published evidence}

To the best of our knowledge current meta-analysis is the only study which provides most reliable and the strongest evidence so far, about the efficacy of SILS against MILS due to strict inclusion criteria evaluating RCTs only. Previously published six systematic reviews (40-42,55-57) lack conclusive outcomes and recommendations due to several 
methodological and clinical flaws such as combined analysis of RCTs and non-RCTs, combined analysis of prospective and retrospective study cohorts and reporting analysis of single colorectal procedure only. Most of these reviews reported feasibility and the safety of the SILS instead providing evidence on either superiority or equivalence of SILS over MILS. Whereas current study conclusively demonstrated that SILS did not show any superiority over MILS for colorectal resections in all post-operative surgical and oncological outcomes.

\section{Implications for practice and research}

Current study conclusively demonstrated that SILS did not show any superiority over MILS for colorectal resections in all post-operative surgical and oncological outcomes. However, long-terms effectiveness still needs to be evaluated. Long-term outcomes in terms of cancer recurrence, survival, incidence of incisional hernia, incidence of port site recurrence, cosmesis and quality of life measurement studies may be required. Studies involving selected cases may show feasibility but all complex colorectal resections may result in opposite outcomes. Current conclusions may well be strengthened by running a major multicentre RCT of high quality. However, wider application of SILS for colorectal resections may not be recommended based upon the findings of current review.

\section{Acknowledgements}

None.

\section{Footnote}

Conflicts of Interest: The abstract of this article was presented as an oral presentation at the Annual General Meeting of the Associations of Surgeons of Great Britain and Ireland in Liverpool on $9^{\text {th }}-11^{\text {th }}$ May 2018. It will be published in the Supplement issue of British fournal of Surgery.

\section{References}

1. Siegel RL, Miller KD, Jemal A. Cancer statistics, 2016. CA Cancer J Clin 2016;66:7-30.

2. Warner E, Crighton EJ, Moineddin R, et al. Fourteenyear study of hospital admissions for diverticular disease in Ontario. Can J Gastroenterol 2007;21:97-9.

3. Painter NS, Burkitt DP. Diverticular disease of the colon: A deficiency disease of Western civilization. BMJ 1971;2:450-4.

4. Lacy AM, García-Valdecasas JC, Delgado S, et al. Laparoscopy assisted colectomy versus open colectomy for treatment of nonmetastatic colon cancer: a randomised trial. Lancet 2002;359:2224-9.

5. Nelson H, Sargent DJ, Wieand HS, et al. Clinical Outcomes of Surgical Therapy Study Group. A comparison of laparoscopically assisted and open colectomy for colon cancer. N Engl J Med 2004;350:2050-9.

6. Guillou PJ, Quirke P, Thorpe H, et al. Short-term endpoints of conventional versus laparoscopic-assisted surgery in patients with colorectal cancer (MRC CLASICC trial): multicentre, randomised controlled trial. Lancet 2005;365:1718-26.

7. Veldkamp R, Kuhry E, Hop WC, et al. Laparoscopic surgery versus open surgery for colon cancer: short-term outcomes of a randomised trial. Lancet Oncol 2005;6:477-84.

8. Fleshman J, Sargent DJ, Green E, et al. Laparoscopic colectomy for cancer is not inferior to open surgery based on 5 -year data from the COST Study Group trial. Ann Surg 2007;246:655-62.

9. Buunen M, Veldkamp R, HopWC, et al. Survival after laparoscopic surgery versus open surgery for colon cancer: long-term outcome of a randomised clinical trial. Lancet Oncol 2009;10:44-52.

10. Schwenk W, Bohm B, Haase O, et al. Laparoscopic versus conventional colorectal resection: A prospective randomized study of postoperative ileus and early postoperative feeding. Langenbecks Arch Surg 1998;383:49-55.

11. Milsom JW, Bohm B, Hammerhofer KA, et al. A prospective, randomized trial comparing laparoscopic versus conventional techniques in colorectal cancer surgery: A preliminary report. J Am Coll Surg 1998;187:46-54; discussion 54-5.

12. Lacy AM, Garcia-Valdecasas JC, Pique JM, et al. Shortterm outcome analysis of a randomized study comparing laparoscopic vs open colectomy for colon cancer. Surg Endosc 1995;9:1101-5.

13. Jayne DG, Guillou PJ, Thorpe H, et al. Randomized trial of laparoscopic-assisted resection of colorectal carcinoma: 3-year results of the UK MRC CLASICC Trial Group. J Clin Oncol 2007;25:3061-8.

14. Laurent C, Leblanc F, Wütrich P, et al. Laparoscopic versus open surgery for rectal cancer: long-term oncologic results. Ann Surg 2009;250:54-61.

15. Schwenk W, Haase O, Neudecker J, et al. Short term benefits for laparoscopic colorectal resection. Cochrane Database Syst Rev 2005;(3):CD003145. 
16. Bonjer HJ, Hop WC, Nelson H, et al. Laparoscopically assisted vs open colectomy for colon cancer: a metaanalysis. Arch Surg 2007;142:298-303.

17. Kang BM, Park SJ, Lee KY, et al. Single-port laparoscopic surgery can be performed safely and appropriately for colon cancer: Short-term results of a pilot Randomized Controlled Trial. J Laparoendosc Adv Surg Tech A 2017;27:501-9.

18. Ahmad G, O'Flynn H, Duffy JM, et al. Laparoscopic entry techniques. Cochrane Database Syst Rev 2008;(2):CD006583.

19. Lee SW, Milsom JW, Nash GM. Single-incision versus multiport laparoscopic right and hand-assisted left colectomy: a case-matched comparison. Dis Colon Rectum 2011;54:1355-61.

20. Arezzo A, Scozzari G, Famiglietti F, et al. Is single-incision laparoscopic cholecystectomy safe? Results of a systematic review and meta-analysis. Surg Endosc 2013;27:2293-304.

21. Antoniou SA, Koch OO, Antoniou GA, et al. Meta-analysis of randomized trials on single-incision laparoscopic versus conventional laparoscopic appendectomy. Am J Surg 2014;207:613-22.

22. Poon JTC, Cheung CW, Fan JKM, et al. Single-incision versus conventional laparoscopic colectomy for colonic neoplasm: a randomized, controlled trial. Surg Endosc 2012;26:2729-34.

23. Champagne BJ, Lee EC, Leblanc F, et al. Single-incision vs straight laparoscopic segmental colectomy: a casecontrolled study. Dis Colon Rectum 2011;54:183-6.

24. Chen WT, Chang SC, Chiang HC, et al. Single-incision laparoscopic versus conventional laparoscopic right hemicolectomy: a comparison of short-term surgical results. Surg Endosc 2011;25:1887-92.

25. Kim SJ, Ryu GO, Choi BJ, et al. The short-term outcomes of conventional and single-port laparoscopic surgery for colorectal cancer. Ann Surg 2011;254:933-40.

26. Papaconstantinou HT, Thomas JS. Single-incision laparoscopic colectomy for cancer: assessment of oncologic resection and short-term outcomes in a casematched comparison with standard laparoscopy. Surgery 2011;150:820-7.

27. Champagne BJ, Papaconstantinou HT, Parmar SS, et al. Single-incision versus standard multiport laparoscopic colectomy: a multicenter, case-controlled comparison. Ann Surg 2012;255:66-9.

28. Lu CC, Lin SE, Chung KC, et al. Comparison of clinical outcome of single-incision laparoscopic surgery using a simplified access system with conventional laparoscopic surgery for malignant colorectal disease. Colorectal Dis 2012;14:e171-6.

29. Ramos-Valadez DI, Ragupathi M, Nieto J, et al. Singleincision versus conventional laparoscopic sigmoid colectomy: a case-matched series. Surg Endosc 2012;26:96-102.

30. Velthuis S, van den Boezem PB, Lips DJ, et al. Comparison of short-term surgical outcomes after singleincision laparoscopic versus multiport laparoscopic right colectomy: a two-center, prospective case-controlled study of 100 patients. Dig Surg 2012;29:477-83.

31. Takemasa I, Uemura M, Nishimura J, et al. Feasibility of single-site laparoscopic colectomy with complete mesocolic excision for colon cancer: a prospective casecontrol comparison. Surg Endosc 2014;28:1110-8.

32. Leroy J, Cahill RA, Asakuma M, et al. Single-access laparoscopic sigmoidectomy as definitive surgical management of prior diverticulitis in a human patient. Arch Surg 2009;144:173-9.

33. Remzi FH, Kirat HT, Kaouk JH, et al. Single-port laparoscopy in colorectal surgery. Colorectal Dis 2008;10:823-6.

34. Boone BA, Wagner P, Ganchuk E, et al. Single-incision laparoscopic right colectomy in an unselected patient population. Surg Endosc 2012;26:1595-601.

35. Katsuno G, Fukunaga M, Nagakari K, et al. Single-incision laparoscopic colectomy for colon cancer: early experience with 31 cases. Dis Colon Rectum 2011;54:705-10.

36. Bucher P, Pugin F, Morel P. Single port access laparoscopic right hemicolectomy. Int J Colorectal Dis 2008;23:1013-6.

37. Huscher CG, Mingoli A, Sgarzini G, et al. Standard laparoscopic versus single-incision laparoscopic colectomy for cancer: early results of a randomized prospective study. Am J Surg 2012;204:115-20.

38. Chew MH, Wong MT, Lim BY, et al. Evaluation of current devices in single-incision laparoscopic colorectal surgery: a preliminary experience in 32 consecutive cases. World J Surg 2011;35:873-80.

39. Yang TX, Chua TC. Single-incision laparoscopic colectomy versus conventional multiport laparoscopic colectomy: a meta-analysis of comparative studies. Int J Colorectal Dis 2013;28:89-101.

40. Luján JA, Soriano MT, Abrisqueta J, et al. Singleport colectomy vs multi-port laparoscopic colectomy. Systematic review and meta-analysis of more than 2800 procedures. Cir Esp 2015;93:307-19.

41. Hoyuela C, Juvany M, Carvajal F. Single-incision laparoscopy versus standard laparoscopy for colorectal surgery: A systematic review and meta-analysis. Am J Surg 
2017;214:127-40.

42. Fung AKY, Aly EH. Systematic review of single-incision laparoscopic colonic surgery. Br J Surg 2012;99:1353-64.

43. Jadad AR, Moore RA, Carroll D, et al. Assessing the quality of reports of randomized clinical trials: is blinding necessary? Control Clin Trials 1996;17:1-12.

44. Chalmers TC, Smith H, Blackburn B, et al. A method for assessing the quality of a randomized control trial. Control Clin Trials 1981;2:31-49.

45. Cochrane IMS. Accessed on 10/03/2018. Available online: http://ims.cochrane.org/revman/otherresources/gradepro/ download

46. Higgins JPT, Green S. editors. Cochrane Handbook for Systematic Reviews of Interventions. Accessed on 10/03/2018. Available online: http://www.cochranehandbook.org

47. Review Manager (RevMan) [Computer program]. Version 5.0. The Nordic Cochrane Centre, The Cochrane Collaboration: Copenhagen, 2008. Available online: http:// tech.cochrane.org/revman/download/windows64. Accessed on $10 / 03 / 2018$.

48. DerSimonian R, Laird N. Meta-analysis in clinical trials. Control Clin Trials 1986;7:177-88.

49. Demets DL. Methods for combining randomized clinical trials: strengths and limitations. Stat Med 1987;6:341-50.

50. Higgins JP, Thompson SG. Quantifying heterogeneity in a meta-analysis. Stat Med 2002;21:1539-58.

51. Egger M, Smith GD, Altman DG. Systematic reviews

doi: $10.21037 / \operatorname{tgh} .2018 .05 .05$

Cite this article as: Hebbar M, Riaz W, Sains P, Baig MK, Sajid MS. Meta-analysis of randomized controlled trials only exploring the role of single incision laparoscopic surgery versus conventional multiport laparoscopic surgery for colorectal resections. Transl Gastroenterol Hepatol 2018;3:30. in healthcare. BMJ Publishing, London, 2006. Available online: http://eu.wiley.com/WileyCDA/WileyTitle/ productCd-072791488X.html. Accessed on 10/03/2018.

52. Deeks JJ, Altman DG, Bradburn MJ. Statistical methods for examining heterogeneity and combining results from several studies in meta-analysis. In: Egger M, Smith GD, Altman DG. editors. Systematic reviews in health care: meta-analysis in context. 2nd edn. London: BMJ Books, 2001:285-312.

53. Kang BM, Kim HJ, Kye BH, et al. Multicenter, randomized single-port versus multiport laparoscopic surgery (SIMPLE) trial in colon cancer: an interim analysis. Surg Endosc 2018;32:1540-9.

54. Watanabe J, Ota M, Fujii S, et al. Randomized clinical trial of single-incision versus multiport laparoscopic colectomy. Br J Surg 2016;103:1276-81.

55. Lolle I, Rosenstock S, Bulut O. Single-port laparoscopic rectal surgery - a systematic review. Dan Med J 2014;61:A4878.

56. Brockhaus AC, Sauerland S, Saad S. Single-incision versus standard multi-incision laparoscopic colectomy in patients with malignant or benign colonic disease: a systematic review, meta-analysis and assessment of the evidence. BMC Surg 2016;16:71.

57. Vettoretto N, Cirocchi R, Randolph J, et al. Single incision laparoscopic right colectomy: a systematic review and meta-analysis. Colorectal Dis 2014;16:O123-32. 